\title{
Síntese e caracterização de zeólita tipo sodalita obtida a partir de resíduo de caulim
}

\author{
(Synthesis and characterization of sodalite-type zeolite \\ obtained from kaolin waste)
}

\author{
B.B.Sousa ${ }^{1 *}$, J.A.R.Rego ${ }^{2}$,D.S.B. Brasil ${ }^{1,3}$, M.C.Martelli ${ }^{1,3}$ \\ ${ }^{I}$ Universidade Federal do Pará, Programa de Pós-Graduação em Engenharia Química, Belém, PA, Brasil \\ ${ }^{2}$ Universidade Federal do Pará, Instituto de Ciências Exatas e Naturais, \\ Faculdade de Ciências Naturais, Belém, PA, Brasil \\ ${ }^{3}$ Universidade Federal do Pará, Instituto de Tecnologia, Faculdade de Engenharia Química, Belém, PA, Brasil
}

\begin{abstract}
Resumo
Produtos zeolíticos foram sintetizados a partir de um resíduo industrial do processo de beneficiamento de caulim. O material de partida foi submetido a tratamento térmico a $700{ }^{\circ} \mathrm{C}$ durante $2 \mathrm{~h}$. As sínteses foram realizadas com a reação do resíduo com $\mathrm{NaOH}$ e uma fonte adicional de sílica, em condições hidrotermais, para relações molares de $\mathrm{SiO}_{2} / \mathrm{Al}_{2} \mathrm{O}_{3}$ de 2 a 4, tempos de cristalização de 24 a 72 h e temperaturas de 90 a $120^{\circ} \mathrm{C}$. A matéria-prima inicial foi caracterizada por FRX, DRX, MEV e TG/DSC, e os produtos sintetizados por meio de DRX, MEV, TG/DSC, FTIR, área específica BET e distribuição de diâmetro de poros. O ensaio realizado a $90{ }^{\circ} \mathrm{C}$, com $48 \mathrm{~h}$ de cristalização e relação $\mathrm{SiO}_{2} / \mathrm{Al}_{2} \mathrm{O}_{3}$ igual a 3 revelou melhor resultado com a formação de sodalita como única fase cristalina. A partir das técnicas de caracterização, foi possível confirmar a formação da sodalita, observando-se os principais aspectos referentes a essa zeólita.

Palavras-chave: zeólita, sodalita, resíduo de caulim.
\end{abstract}

\section{Abstract}

Zeolite products were synthesized from industrial waste from kaolin processing. The starting material was submitted to thermal treatment at $700{ }^{\circ} \mathrm{C}$ for $2 \mathrm{~h}$. Synthesis processes were carried out by reacting kaolin waste with $\mathrm{NaOH}$ and an additional source of silica, in hydrothermal conditions, for $\mathrm{SiO}_{2} / \mathrm{Al}_{2} \mathrm{O}_{3}$ molar ratios from 2 to 4 , for 24 to $72 \mathrm{~h}$ of crystallization at 90 to $120{ }^{\circ} \mathrm{C}$. The starting material was characterized by XRF, XRD, SEM, and TG/DSC, and the synthesized products were characterized by XRD, $S E M, X R F, T G / D S C$, FTIR, BET surface area, and pore diameter distribution. The product obtained at $90{ }^{\circ} \mathrm{C}$ for $48 \mathrm{~h}$ using the $\mathrm{SiO}_{2} / \mathrm{Al}_{2} \mathrm{O}_{3}$ ratio of 3 showed the best result, forming sodalite as the only crystalline phase. From the characterization techniques, it was possible to confirm the formation of sodalite, observing the main aspects related to this zeolite.

Keywords: zeolite, sodalite, kaolin waste.

\section{INTRODUÇÃO}

O estado do Pará se destaca dentro do cenário nacional por possuir um dos mais importantes depósitos de caulim a nível internacional e por ser o principal distrito brasileiro de produção de caulim para a indústria de papel [1,2]. Como o caulim ocorre associado a diversas impurezas, torna-se necessário a realização de processos de beneficiamento, a fim de adequá-lo às especificações de mercado e aplicações industriais. No ano de 2016, a produção beneficiada foi cerca de 1,6 milhão de toneladas [3]. O processamento mais utilizado é o beneficiamento a úmido, o qual envolve as etapas de dispersão, desareamento, fracionamento em hidrociclone ou centrífuga, separação magnética, floculação

*brunabragasousa@gmail.com

Dhttps://orcid.org/0000-0002-3662-9770 seletiva, alvejamento químico, filtragem e secagem [1, 2]. Nesse sentido, o beneficiamento do caulim é responsável por gerar grandes volumes de resíduos, cerca de 1,2 milhão de toneladas por ano [3]. Um tipo de resíduo é composto por partículas grosseiras, basicamente constituídas de quartzo proveniente da etapa de desaeramento, e que são repostas no próprio local da lavra. Outro resíduo proveniente de outras etapas, constituído predominantemente por caulinita, é armazenado em grandes lagoas de sedimentação, o que gera problemas tanto de ordem financeira como ambiental $[2,4,5]$. O caulim, além de ser abundante e de baixo custo, representa uma matéria-prima interessante na realização de sínteses de zeólitas [4]. Isso se deve sobretudo ao fato da composição química deste material (predominantemente caulinita) ser semelhante à composição de materiais zeolíticos. Dessa forma, a investigação do resíduo proveniente do processo de beneficiamento do caulim como precursor na obtenção de 
zeólitas torna-se um estudo relevante, como uma alternativa para o reaproveitamento deste material [3-5].

As zeólitas são aluminossilicatos hidratados cristalinos de elementos dos grupos I e II da tabela periódica, em particular sódio, potássio, magnésio, cálcio, estrôncio e bário. Atualmente este conceito pode ser expandido a fim de incluir um grande número de materiais que possuem estruturas semelhantes e que apresentam em sua composição outros cátions além do $\mathrm{Si} \mathrm{eAl}$, tais como B, Ge, Fe, P, Co, Zn, etc. $[4,6,7]$. A estrutura das zeólitas consiste em uma rede tridimensional infinitamente extensa de tetraedros de $\left[\mathrm{AlO}_{4}\right]^{5-}$ e $\left[\mathrm{SiO}_{4}\right]^{4-}$ ligados entre si por oxigênios compartilhados. A substituição de um átomo de $\mathrm{Si}^{4+}$ por um átomo de $\mathrm{Al}^{3+}$ leva a uma estrutura com carga negativa na superfície, sendo necessária a presença de um íon positivo para compensar a substituição isomórfica. Esses cátions possuem mobilidade, resultando em alta capacidade de troca iônica $[4,6]$. As principais aplicações envolvendo o uso de zeólitas estão no uso em adsorção e catálise. Além disso, as zeólitas naturais são amplamente utilizadas em aplicações minerais [6]. Esses materiais também podem ser sintetizados em grande escala para usos específicos ou obtidos em laboratório, a fim de investigar a estrutura e as propriedades dos cristais. Dessa forma, podem ser modificados com o intuito de gerar uma variedade de produtos, com propriedades específicas para uso industrial [8]. Zeólitas sintéticas normalmente são produzidas a partir de géis de silicato e aluminato de sódio, utilizando reagentes comerciais. Entretanto, estudos vêm sendo realizados na síntese a partir de matérias-primas naturais. Nesse sentido, o caulim se apresenta como uma matéria-prima interessante na síntese de zeólitas de baixa razão molar sílica-alumina, devido às razões molares de $\mathrm{SiO}_{2} / \mathrm{Al}_{2} \mathrm{O}_{3}$ serem bastante semelhantes (aproximadamente iguais a 2). Pesquisas envolvendo esse material atingiram resultados favoráveis [8-11]. A caulinita pode ser modificada estruturalmente através de tratamento térmico (calcinação) a cerca de $600-700{ }^{\circ} \mathrm{C}$. A nova fase formada, amorfa aos raios $\mathrm{X}$, é chamada de metacaulinita. Esta fase apresenta silício e alumínio mais livres, tornando o material mais reativo e adequado para a síntese de zeólitas. O material pode então ser tratado com soluções aquosas de hidróxido de metal alcalino para a síntese, sendo o produto formado relacionado com a composição da mistura reacional [3].

$\mathrm{O}$ mineral sodalita, com composição química $\mathrm{Na}_{8}\left(\mathrm{AlSiO}_{4}\right)_{6} \mathrm{Cl}_{2}$, foi descoberto por Thomson [12] e mais tarde teve sua estrutura descrita por Pauling [13]. Desde então, o aprimoramento nas pesquisas com o desenvolvimento de novos métodos de síntese permitiu a obtenção de sodalitas com composições químicas variadas. De acordo com o tipo de íons ou moléculas presentes na estrutura, sodalitas com diferentes características químicas são descritas e podem ser classificadas. Um membro interessante da família da sodalita é a sodalita hidratada (ou hidroxisodalita), $\mathrm{Na}_{8}\left(\mathrm{AlSiO}_{4}\right)_{6}(\mathrm{OH})_{2} \cdot \mathrm{nH}_{2} \mathrm{O}, 0<\mathrm{n} \leq 4$, sintetizada em condições hidrotermais $[14,15]$. $\mathrm{O}$ arranjo estrutural da sodalita é baseado em uma série de poliedros octaédricos regulares, chamados de cavidade sodalita ou $\beta$-cavidade. A cavidade é formada por 6 anéis simples de 4 tetraedros e 8 anéis simples de 6 tetraedros. A união dessas subunidades forma a estrutura da sodalita, com canais e cavidades interconectados, possuindo anéis de 6 membros com diâmetro cinético de abertura de 2,65 Å. Dessa forma, apenas moléculas muito pequenas, tais como hélio $(2,6$ $\AA$ ), amônia $(2,5 \AA$ ) e água $(2,65 \AA)$, podem acessar os canais e cavidades, tornando a sodalita uma candidata em potencial na separação de moléculas pequenas $[4,16]$. A maioria das pesquisas encontradas na literatura obtém a sodalita em mistura com outras fases [17-20]; portanto, a síntese da sodalita pura representa um estudo de grande relevância, justamente pela alta seletividade para moléculas pequenas que a zeólita apresenta. Neste trabalho, zeólita do tipo sodalita foi sintetizada em condições hidrotermais, utilizando resíduo de caulim como material de partida, a fim de propor uma forma de reaproveitamento desse resíduo.

\section{EXPERIMENTAL}

Materiais: como fonte de silício e alumínio utilizouse um resíduo caulinítico obtido como subproduto do processo de beneficiamento de caulim, proveniente de uma empresa localizada na região do Rio Capim, no estado do Pará, Brasil. O material foi previamente seco e peneirado em peneira de 100 mesh $(<0,149 \mathrm{~mm})$ para a realização dos ensaios de síntese. Os reagentes analíticos utilizados foram: hidróxido de sódio em micropérola P.A. (Neon), com teor de $97 \%$ em $\mathrm{NaOH}$, usado como fonte de metal alcalino ao processo; sílica gel 60 (Merck), com volume de poros de 0,74-0,84 mL/g, área específica de $480-540 \mathrm{~m}^{2} / \mathrm{g}$ e distribuição granulométrica na faixa de 75 a $245 \mu \mathrm{m}$, utilizada como fonte secundária de silício; e, como solvente, utilizou-se água destilada.

Ensaios de síntese: o resíduo caulinítico foi inicialmente submetido a tratamento térmico a $700{ }^{\circ} \mathrm{C}$ por $2 \mathrm{~h}$ em forno mufla (metacaulinização) a fim de promover a desestruturação cristalina do material. Esses valores foram baseados em resultados da literatura [8, 9], os quais se mostraram adequados na formação da metacaulinita. Os ensaios de síntese foram realizados em condições hidrotermais em autoclave de aço inoxidável revestida internamente por

Tabela I - Condições de síntese.

[Table I - Synthesis conditions.]

\begin{tabular}{cccc}
\hline Ensaio & $\mathrm{SiO}_{2} / \mathrm{Al}_{2} \mathrm{O}_{3}$ & $\begin{array}{c}\text { Tempo } \\
(\mathrm{h})\end{array}$ & $\begin{array}{c}\text { Temperatura } \\
\left({ }^{\circ} \mathrm{C}\right)\end{array}$ \\
\hline $1^{\mathrm{a}}$ & 2,0 & 24 & 90 \\
$2^{\mathrm{a}}$ & 3,0 & 24 & 90 \\
$3^{\mathrm{a}}$ & 4,0 & 24 & 90 \\
$4^{\mathrm{b}}$ & 3,0 & 48 & 90 \\
$5^{\mathrm{b}}$ & 3,0 & 72 & 90 \\
$6^{\mathrm{c}}$ & 3,0 & 48 & 120 \\
\hline
\end{tabular}

${ }^{a}$ influência da relação $\mathrm{SiO}_{2} / \mathrm{Al}_{2} \mathrm{O}_{3},{ }^{b}$ influência do tempo; ${ }^{c}$ influência da temperatura. 
um vaso de teflon e capacidade de $40 \mathrm{~mL}$. A autoclave foi fechada e mantida em repouso em estufa a temperaturas e tempos determinados. Ao término das sínteses, a autoclave foi retirada e resfriada em água corrente até a temperatura ambiente. Os produtos formados foram filtrados com papel de filtro quantitativo e lavados com água destilada até $\mathrm{pH}$ $\sim$, a fim de evitar excesso de alcalinidade. Em seguida, os materiais filtrados foram secos em estufa a $90{ }^{\circ} \mathrm{C}$ por $24 \mathrm{~h}$, pulverizados e armazenados para posterior caracterização. As quantidades das matérias-primas para o gel de síntese foram calculadas estequiometricamente baseadas na composição molar $7 \mathrm{Na}_{2} \mathrm{O}: \mathrm{Al}_{2} \mathrm{O}_{3}: \mathrm{xSiO}_{2}: \mathrm{H}_{2} \mathrm{O}$, variando-se os valores de $\mathrm{x}$, tempo e temperatura de cristalização, para um volume total de água de aproximadamente $30 \mathrm{~mL}$. Na Tabela I são encontradas as condições utilizadas nas sínteses dos materiais zeolíticos, tomando como base $3 \mathrm{~g}$ de metacaulim.

Fluorescência de raios $X(F R X)$ : a determinação da composição química das amostras por FRX foi realizada utilizando espectrômetro por dispersão de comprimento de onda (WDS) sequencial (Axios Minerals, PANalytical), com tubo de raios $X$ cerâmico, anodo de ródio $(\mathrm{Rh})$ e máximo nível de potência de $2,4 \mathrm{~kW}$. As aquisições e tratamento dos dados foram realizados através do software SuperQ Manager (PANalytical). A amostra foi preparada por pastilha prensada: $0,5 \mathrm{~g}$ de amostra mais $0,15 \mathrm{~g}$ de aglomerante (cera de parafina) sobre substrato de ácido bórico $\left(\mathrm{H}_{3} \mathrm{BO}_{3}\right)$. A mistura foi prensada com carga de 25 ton, diâmetro da pastilha de $33 \mathrm{~mm}$ e espessura de $3 \mathrm{~mm}$. Difração de raios $X(D R X)$ : para identificar as principais fases cristalinas do resíduo de caulim e metacaulim, assim como dos produtos sintetizados, a caracterização por DRX foi realizada usando um difratômetro (D8 Advance, Bruker) com goniômetro $\theta / \theta$ e tubo de raios $X$ cerâmico de ânodo de $\mathrm{Cu}(\mathrm{CuK} \alpha 1=1,54058 \AA)$, mod. 10190376, a 2,2 kW/60 $\mathrm{kV}$. O detector utilizado foi o Lynxeye e a varredura foi realizada na faixa de $5^{\circ}$ a $75^{\circ}(2 \theta)$. Microscopia eletrônica de varredura $(M E V)$ : para observar a morfologia dos materiais, foi utilizado um microscópio eletrônico (Mira3, Tescan) com canhão de elétrons tipo FEG (field emission gun). As amostras foram montadas em suportes de alumínio com $12 \mathrm{~mm}$ de diâmetro utilizando-se fita adesiva dupla face de carbono e metalizadas com Au por 1,5 min, o que depositou sobre a amostra uma película com espessura média de 10 a $15 \mathrm{~nm}$. As imagens foram geradas por detecção de elétrons secundários, utilizando-se aceleração de voltagem de $5 \mathrm{kV}$ e distância de trabalho entre 10 e $15 \mathrm{~mm}$. Análise térmica (TG/DSC): análises de termogravimetria (TG) e calorimetria exploratória diferencial (DSC) foram realizadas em equipamento (STA 449F5, Netzsch) com analisador térmico simultâneo e conversor digital acoplado a um microcomputador, utilizando cadinho de alumina com cerca de $30 \mathrm{mg}$ de amostra, com varredura na faixa de temperatura ambiente até $1100{ }^{\circ} \mathrm{C}$ e taxa de aquecimento de $20^{\circ} \mathrm{C} \cdot \mathrm{min}^{-1}$. Espectroscopia no infravermelho com transformada de Fourier (FTIR): a análise por FTIR foi realizada em equipamento (IR Prestige-21, Shimadzu) com varredura de número de onda na região de 4000 a $400 \mathrm{~cm}^{-1}$, resolução de $2 \mathrm{~cm}^{-1}$ e pastilhas produzidas com $\mathrm{KBr}$. Caracterização textural por fisissorção de nitrogênio: isotermas de adsorção e dessorção de nitrogênio foram obtidas na temperatura de ebulição do nitrogênio (Tristar II Kr 3020, Micromeritics). A amostra foi previamente aquecida a $120{ }^{\circ} \mathrm{C}$ sob vácuo por $12 \mathrm{~h}$. As áreas específicas foram estimadas usando-se o método BET (Brunauer-Emmett-Teller) e a distribuição de tamanho de poros usando-se o método DFT (density functional theory).

\section{RESULTADOS E DISCUSSÃO}

\section{Material de partida}

Os resultados de FRX para o resíduo de caulim, em comparação com a composição nominal da caulinita, estão apresentados na Tabela II. Observou-se que os percentuais de $\mathrm{SiO}_{2}(47,30 \%), \mathrm{Al}_{2} \mathrm{O}_{3}(37,00 \%)$ e perda ao fogo $(13,84 \%)$ do resíduo caulinítico foram próximos aos valores apresentados pela composição nominal da caulinita, indicando que o material foi essencialmente constituído pelo argilomineral caulinita. As pequenas diferenças foram atribuídas à presença de impurezas, as quais somaram $1,86 \%$. Vale ressaltar que o material caulinítico utilizado foi de origem sedimentar. É importante pontuar que, além da sílica proveniente da caulinita, o teor total de $\mathrm{SiO}_{2}$ também leva em consideração a presença da sílica relativa ao quartzo, porém em pequena quantidade, como mostrado pela difração de raios X (Fig. 1a). As impurezas encontradas no resíduo foram óxidos de ferro $\left(\mathrm{Fe}_{2} \mathrm{O}_{3}\right)$ e de titânio $\left(\mathrm{TiO}_{2}\right)$, com $1,03 \%$ e $0,83 \%$, respectivamente.

A Fig. 1a apresenta a análise mineralógica do resíduo de caulim a partir de difração de raios $\mathrm{X}$. A análise do difratograma indicou que o resíduo foi constituído essencialmente de caulinita, comprovada pela presença dos picos principais desse mineral (PDF 83-0971) em $2 \theta$ igual a $12,42^{\circ}(7,12 \AA), 20,42^{\circ}(4,35 \AA), 24,96^{\circ}$ (3,57 $\left.\mathrm{A}\right)$ e $21,33^{\circ}$ $(4,16 \AA)$. A partir do difratograma, observou-se a formação dos tripletos que compreenderam os picos em $2 \theta$ igual a $19,94^{\circ}(4,45 \AA), 20,42^{\circ}(4,35 \AA)$ e $21,33^{\circ}(4,16 \AA), 35,03^{\circ}$ $(2,56 \AA), 35,48^{\circ}(2,53 \AA)$ e $35,99^{\circ}(2,50 \AA)$, e $37,77^{\circ}(2,38$ $\AA), 38,51^{\circ}(2,34 \AA)$ e $39,35^{\circ}(2,29 \AA)$, característicos da caulinita [21]. Ocorreu também a presença de quartzo como mineral secundário (PDF 85-0930), indicado pelo pico em

Tabela II - Resultados de FRX do resíduo de caulim. [Table II - XRF results of the kaolin waste.]

\begin{tabular}{ccc}
\hline Óxido & $\begin{array}{c}\text { Resíduo de } \\
\text { caulim (\%) }\end{array}$ & $\begin{array}{c}\text { Composição nominal } \\
\text { da caulinita (\%) }\end{array}$ \\
\hline $\mathrm{SiO}_{2}$ & 47,30 & 46,54 \\
$\mathrm{Al}_{2} \mathrm{O}_{3}$ & 37,00 & 39,50 \\
$\mathrm{Fe}_{2} \mathrm{O}_{3}$ & 1,03 & - \\
$\mathrm{TiO}_{2}$ & 0,83 & - \\
P.F. & 13,84 & 13,96 \\
\hline
\end{tabular}

P.F.: perda ao fogo. 
$2 \theta$ em $26,45^{\circ}$ (3,37 Å). Após o processo de calcinação, observou-se a perda de cristalinidade do resíduo de caulim (Fig. 1b), através do desaparecimento de reflexões fortes e agudas da caulinita, além de uma elevação do background na região entre $20^{\circ}$ e $30^{\circ}$, mostrando a presença de material amorfo, constituído principalmente por sílica [7]. Esse processo é essencial para a desestruturação cristalina do resíduo de caulim, de modo a facilitar a reorganização de um novo produto, em condições específicas [9]. Portanto, o tratamento térmico (metacaulinização) a $700{ }^{\circ} \mathrm{C}$ por $2 \mathrm{~h}$ mostrou-se eficiente para a quebra da estrutura, uma vez que os picos principais e os tripletos da caulinita não apareceram mais na estrutura do metacaulim.
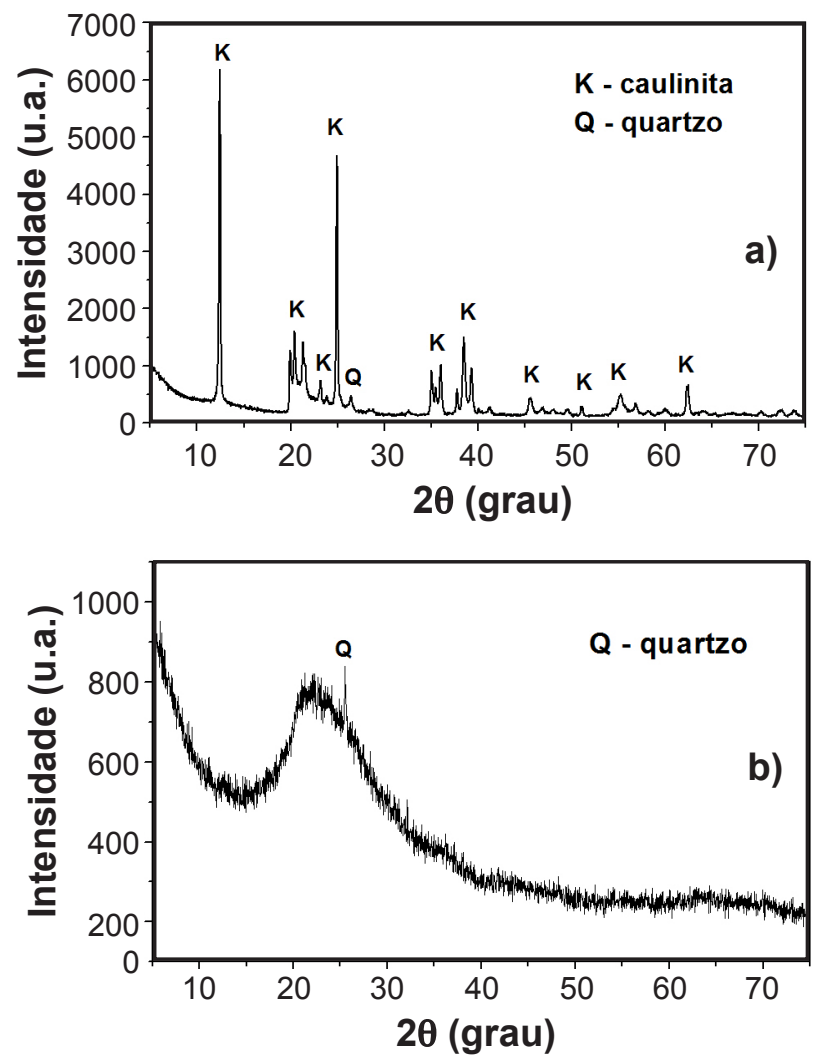

Figura 1: Difratogramas de raios $\mathrm{X}$ do resíduo caulinítico antes (a) e após (b) calcinação por $2 \mathrm{~h}$ a $700{ }^{\circ} \mathrm{C}$.

[Figure 1: XRD patterns of the kaolin waste before (a) and after (b) calcination for $2 \mathrm{~h}$ at $700{ }^{\circ} \mathrm{C}$.]

A Fig. 2 apresenta as imagens de MEV para o resíduo de caulim (Fig. 2a) e metacaulim (Fig. 2b). Observou-se que o resíduo de caulim foi constituído em maior parte por partículas aglomeradas com empilhamento do tipo livreto, com cristais de morfologia pseudo-hexagonal característicos da caulinita [5]. Por outro lado, o caulim calcinado (metacaulim), apesar de também apresentar aglomerados com a mesma morfologia do resíduo sem tratamento, mostrou alteração do empilhamento do tipo livreto, com a diminuição das folhas empacotadas, confirmando o processo de desestruturação [5, 7, 17, 21].

A Fig. 3 apresenta os resultados de TG/DSC mostrando as modificações de massa que ocorreram com a amostra do

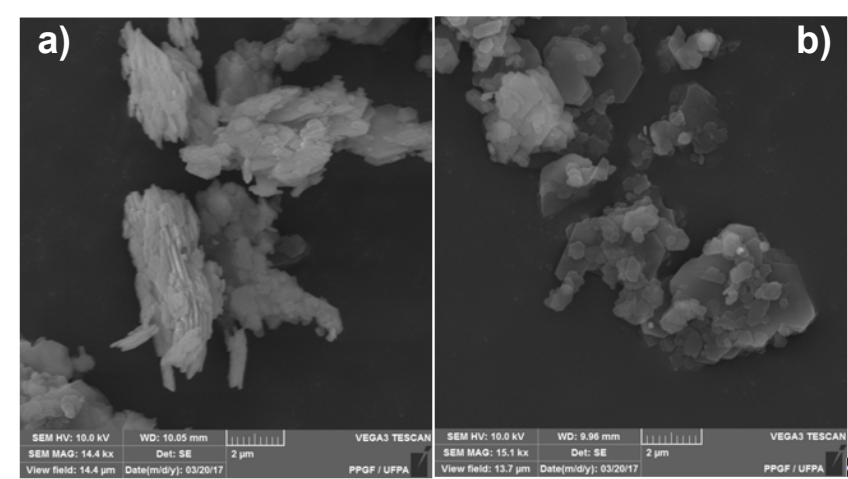

Figura 2: Imagens de MEV do resíduo de caulim antes (a) e após (b) calcinação por $2 \mathrm{~h}$ a $700{ }^{\circ} \mathrm{C}$.

[Figure 2: SEM images of kaolin waste before (a) and after (b) calcination for 2 h at $700{ }^{\circ} \mathrm{C}$.]

resíduo de caulim em função da elevação da temperatura. A análise de DSC revelou um evento endotérmico com máximo em $514{ }^{\circ} \mathrm{C}$, referente à desidroxilação da caulinita e consequente transformação em metacaulinita, e um evento exotérmico em torno de $986{ }^{\circ} \mathrm{C}$ devido à decomposição da metacaulinita e cristalização da fase mulita ou espinélio alumínio-silício [22]. Pela análise termogravimétrica, observou-se que a perda de massa na amostra ocorreu principalmente em torno de $500{ }^{\circ} \mathrm{C}$, dada pela desidroxilação da caulinita, com uma perda de massa total da amostra de $14,32 \%$ próxima ao valor observado na FRX.

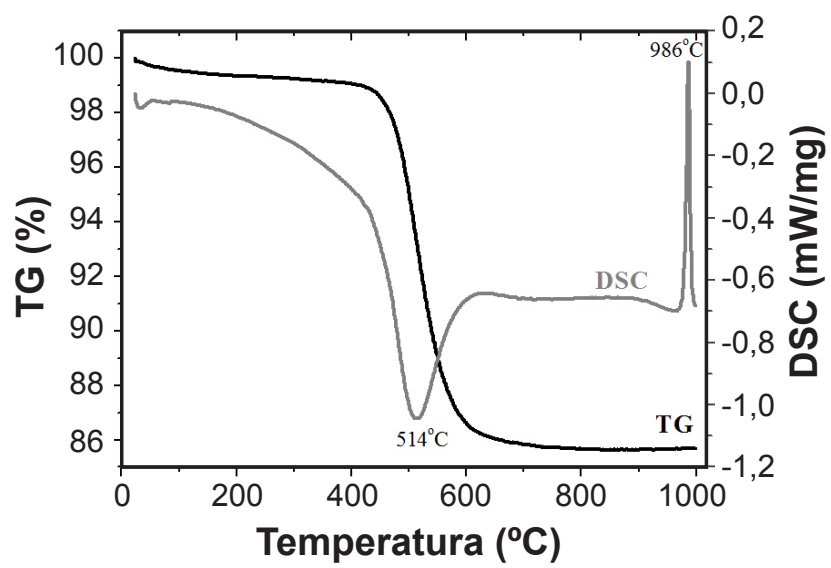

Figura 3: Curvas de TG e DSC do resíduo de caulim.

[Figure 3: TG and DSC curves of the kaolin waste.]

\section{Sintese}

Os efeitos da relação $\mathrm{SiO}_{2} / \mathrm{Al}_{2} \mathrm{O}_{3}$ e do tempo de reação na composição molar da mistura reacional foram analisados, assim como da temperatura de cristalização, procurando-se levar em consideração a ação destas variáveis no processo de formação da fase zeolítica desejada. As informações referentes às fases cristalinas presentes nos produtos sintetizados foram obtidas por meio da técnica de difração de raios $X$.

Influência da relação $\mathrm{SiO}_{2} / \mathrm{Al}_{2} \mathrm{O}_{3}$ : com o objetivo de observar como a relação entre sílica e alumina influencia 
no processo de formação da zeólita do tipo sodalita, foram sintetizadas amostras utilizando relação molar igual a 2, 3 e 4, para um tempo de cristalização de $24 \mathrm{~h}$ e temperatura de $90{ }^{\circ} \mathrm{C}$. Como pode ser visto por meio dos picos de difração de raios X da Fig. 4a, para uma razão molar de $\mathrm{SiO}_{2} / \mathrm{Al}_{2} \mathrm{O}_{3}$ igual a 2 , obteve-se como fase predominante zeólita A no padrão difratométrico (PDF 89-8015), com reflexões intensas dessa fase em $2 \theta$ a igual a $7,18^{\circ}(12,30 \AA)$, $10,17^{\circ}(8,69 \AA), 16,15^{\circ}(5,48 \AA)$ e $12,49^{\circ}(7,08 \AA)$. Como fase concorrente ocorreu a presença da sodalita, mas em pequena proporção, com pico em $13,99^{\circ}(6,32 \AA)$. Esse
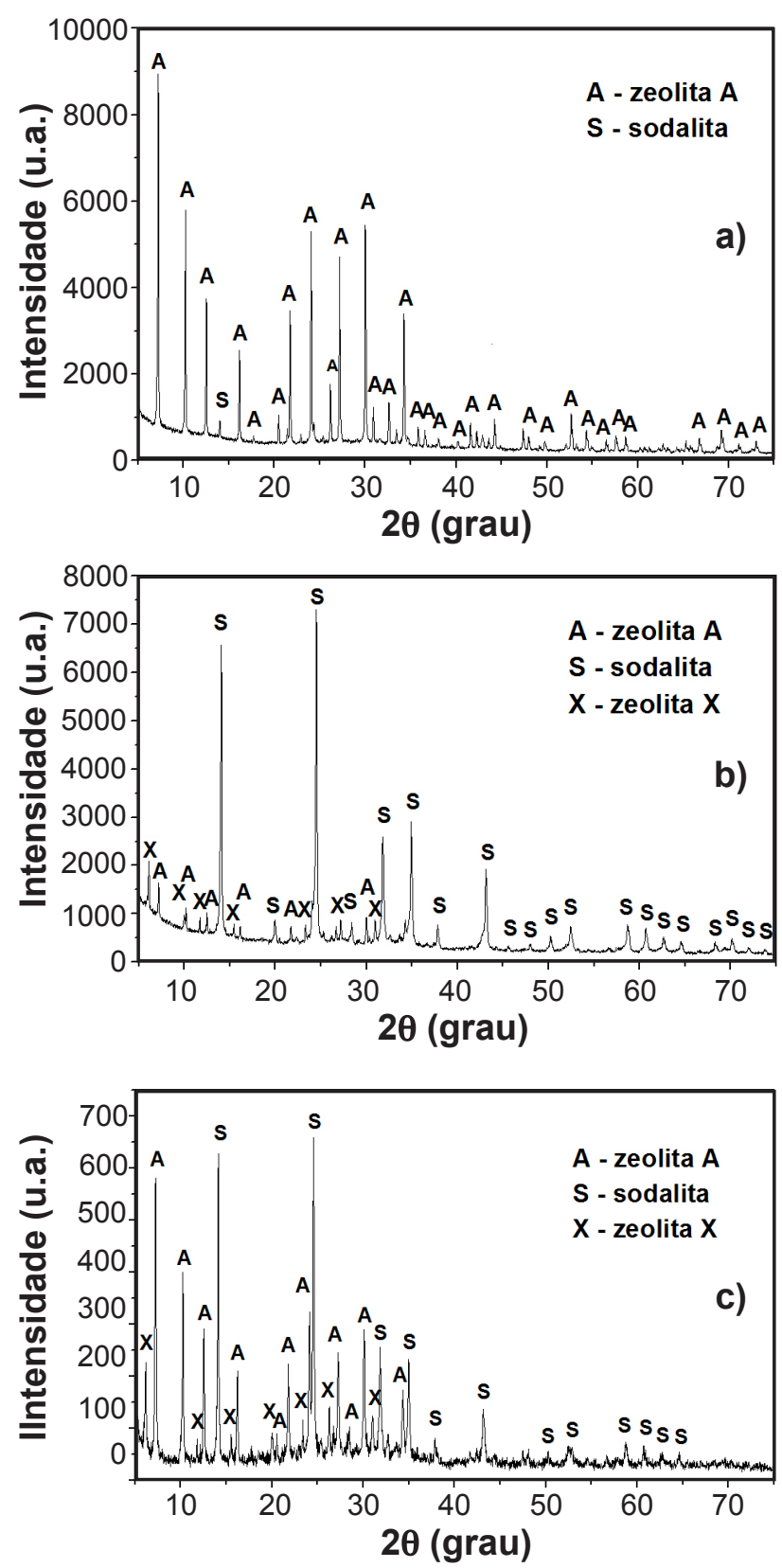

Figura 4: Difratogramas de raios $\mathrm{X}$ dos produtos zeolíticos para cristalização de 24 h a $90{ }^{\circ} \mathrm{C}$ com relação molar $\mathrm{SiO}_{2} / \mathrm{Al}_{2} \mathrm{O}_{3}$ igual a: a) 2 ; b) 3 ; e c) 4 .

[Figure 4: XRD patterns of the zeolitic products obtained for 24 $h$ of crystallization at $90{ }^{\circ} \mathrm{C}$ using $\mathrm{SiO}_{2} / \mathrm{Al}_{2} \mathrm{O}_{3}$ ratio of: a) 2; b) 3; and c) 4.] resultado foi similar ao encontrado na literatura para zeólita A [18]. Observou-se por meio da Fig. 4b que ao aumentar a relação $\mathrm{SiO}_{2} / \mathrm{Al}_{2} \mathrm{O}_{3}$ para 3 ocorreu a formação de zeólita do tipo sodalita (PDF 76-1639) como fase principal, através da presença das reflexões principais do mineral em $2 \theta$ igual a $24,49^{\circ}(3,63 \AA)$ e $14,08^{\circ}(6,29 \AA)$, além de outros picos característicos dessa fase. Para essa proporção, a zeólita A apareceu como fase concorrente, assim como zeólita X (faujasita, PDF 76-1639) com os picos principais em $2 \theta$ igual a $6,10^{\circ}(14,47 \AA), 9,99^{\circ}(8,85 \AA)$, entre outros. Resultados semelhantes são encontrados na literatura [7, 19]. Para uma
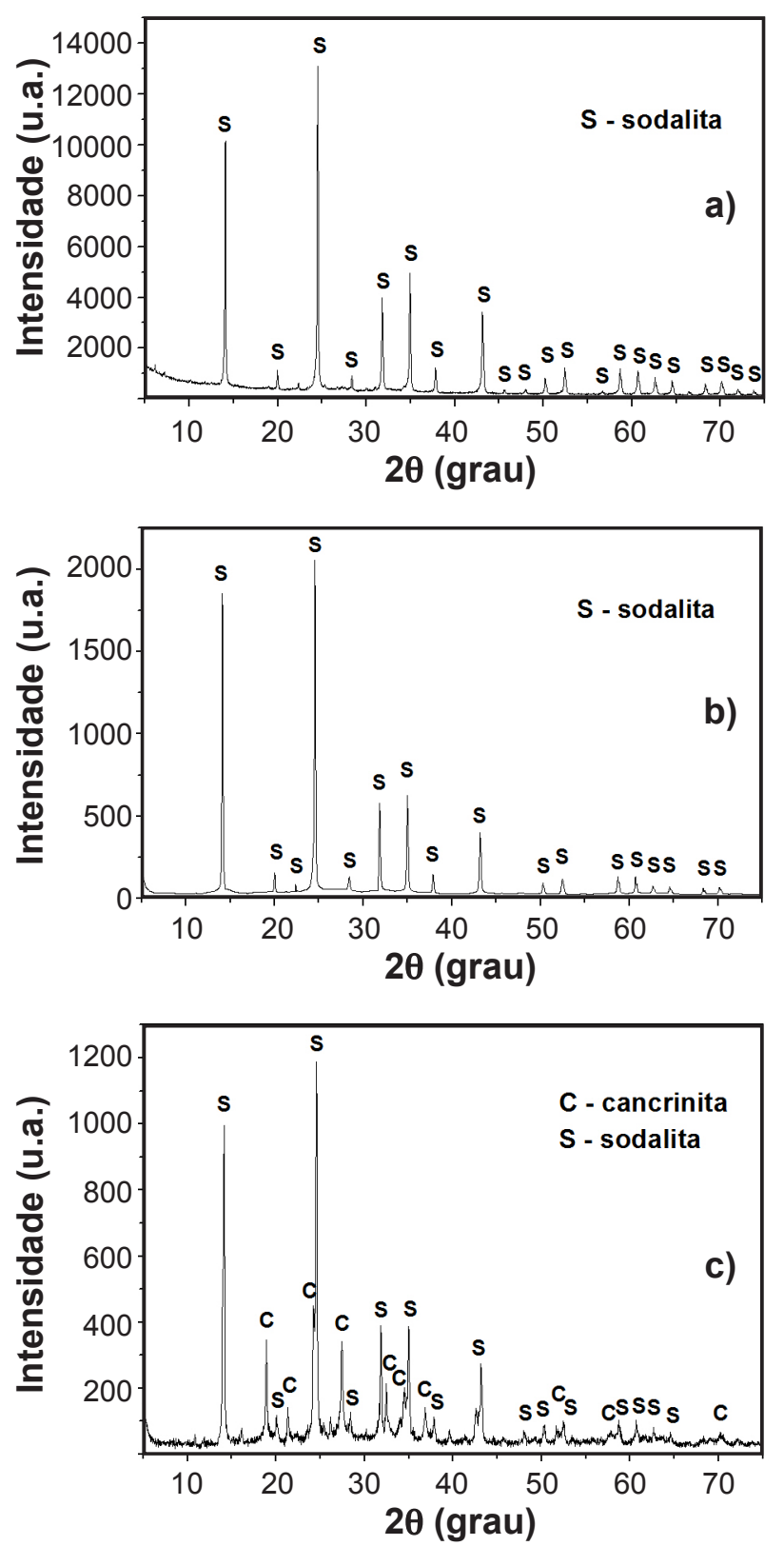

Figura 5: Difratogramas de raios $\mathrm{X}$ dos produtos zeolíticos para cristalização com relação molar $\mathrm{SiO}_{2} / \mathrm{Al}_{2} \mathrm{O}_{3}=3$ nas condições de: a) $48 \mathrm{~h}$ a $90^{\circ} \mathrm{C}$; b) $72 \mathrm{~h}$ a $90{ }^{\circ} \mathrm{C}$; e c) $48 \mathrm{~h}$ a $120^{\circ} \mathrm{C}$.

[Figure 5: XRD patterns of the zeolitic products for crystallization using $\mathrm{SiO}_{2} / \mathrm{Al}_{2} \mathrm{O}_{3}$ ratio of 3 and calcination conditions of: a) $48 \mathrm{~h}$ at $90{ }^{\circ} \mathrm{C}$; b) $72 \mathrm{~h}$ at $90{ }^{\circ} \mathrm{C}$; and c) $48 \mathrm{~h}$ at $120^{\circ} \mathrm{C}$.] 
relação molar igual a 4, observou-se novamente a presença das fases sodalita, zeólita X e zeólita A (Fig. 4c). Estudos analisando a influência de diferentes variáveis no processo de síntese de zeólitas do tipo faujasita também revelaram essa fase em conjunto com sodalita e zeólita A nos produtos zeolíticos obtidos, para composição molar $7 \mathrm{Na}_{2} \mathrm{O}-\mathrm{Al}_{2} \mathrm{O}_{3}$ $3 \mathrm{SiO}_{2}-\mathrm{H}_{2} \mathrm{O}$ e em diferentes tempos de cristalização $[6,7]$.

Influência do tempo de cristalização: uma vez que com a proporção de $\mathrm{SiO}_{2} / \mathrm{Al}_{2} \mathrm{O}_{3}$ igual a 3 o produto obtido já apresentou sodalita como fase principal, observou-se como o tempo de reação poderia influenciar no processo de formação da zeólita do tipo sodalita para ensaios utilizando a mesma proporção, na mesma temperatura de $90{ }^{\circ} \mathrm{C}$. A partir do difratograma da Fig. 5a, para o tempo de cristalização de $48 \mathrm{~h}$, a zeólita sodalita foi formada como fase dominante, com os picos principais em $2 \theta$ igual a $24,49^{\circ}(3,63 \AA)$ e $14,08^{\circ}(6,29 \AA)$, além de outros picos característicos dessa fase, desaparecendo os picos de zeólita A e zeólita X. Para o tempo de 72 h (Fig. 5b), a zeólita sodalita também foi formada como fase dominante, com os picos principais em $2 \theta$ igual a $24,54^{\circ}(3,62 \AA)$ e $14,11^{\circ}(6,27 \AA)$, além de outros picos característicos, novamente não aparecendo picos relativos a outras fases zeolíticas. Estudo realizado com a composição reacional $3 \mathrm{Na}_{2} \mathrm{O}-3 \mathrm{SiO}_{2}-\mathrm{Al}_{2} \mathrm{O}_{3}-90 \mathrm{H}_{2} \mathrm{O}$, mesma relação de sílica/alumina da presente pesquisa, porém em mais baixa alcalinidade, também resultou em sodalita como fase principal ao serem analisados diferentes tempos de cristalização a $95{ }^{\circ} \mathrm{C}$ [19].

Influência da temperatura: a temperatura representa uma variável de síntese com forte efeito na formação das zeólitas. Normalmente o material zeolítico desejado só é obtido dentro de uma faixa específica de temperatura [23]. A fim de se verificar como o aumento da temperatura influencia no processo de cristalização da zeólita sodalita, foi realizado ensaio a $120^{\circ} \mathrm{C}$ utilizando a relação molar $\mathrm{SiO}_{2} / \mathrm{Al}_{2} \mathrm{O}_{3}$ igual a 3 , melhor proporção encontrada. A partir do difratograma da Fig. 5c, o aumento da temperatura de síntese resultou no aparecimento da fase cancrinita (PDF 46-1457) no produto zeolítico como concorrente a sodalita, com os picos principais em $2 \theta$ igual a $27,40^{\circ}(3,25 \AA), 24,23^{\circ}(3,67 \AA), 18,89^{\circ}(4,69$ $\AA$ ) e $36,84^{\circ}(2,44 \AA)$. Em temperaturas mais altas (a partir de $120^{\circ} \mathrm{C}$ ), zeólita do tipo cancrinita pode ser formada em
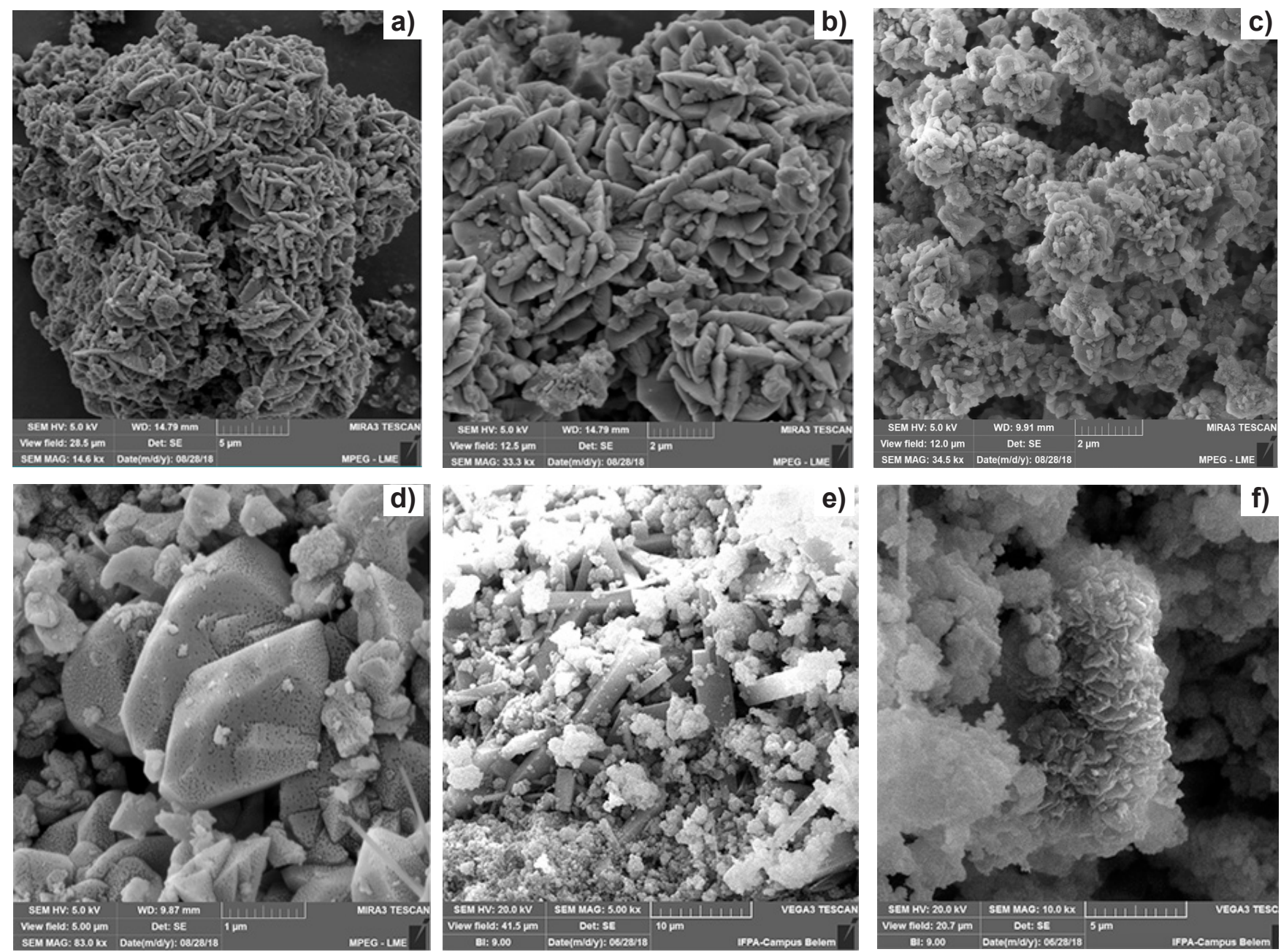

Figura 6: Imagens de MEV dos produtos zeolíticos para cristalização nas condições: a,b) $\mathrm{SiO}_{2} / \mathrm{Al}_{2} \mathrm{O}_{3}=3,48 \mathrm{~h}$ a $\left.90{ }^{\circ} \mathrm{C} ; \mathrm{c}\right) \mathrm{SiO}_{2} / \mathrm{Al}_{2} \mathrm{O}_{3}=3,72 \mathrm{~h}$ a $90{ }^{\circ} \mathrm{C}$; d) $\mathrm{SiO}_{2} / \mathrm{Al}_{2} \mathrm{O}_{3}=4,24 \mathrm{~h}$ a $90{ }^{\circ} \mathrm{C}$; e,f) $\mathrm{SiO}_{2} / \mathrm{Al}_{2} \mathrm{O}_{3}=3,48 \mathrm{~h}$ a $120{ }^{\circ} \mathrm{C}$.

[Figure 6: SEM images of the zeolitic products for crystallization conditions of: a,b) $\mathrm{SiO}_{2} / \mathrm{Al}_{2} \mathrm{O}_{3}=3,48 \mathrm{~h}$ at $\left.90^{\circ} \mathrm{C} ; \mathrm{c}\right) \mathrm{SiO}_{2} / \mathrm{Al}_{2} \mathrm{O}_{3}=3,72 \mathrm{~h}$ at $90{ }^{\circ} \mathrm{C}$; d) $\mathrm{SiO}_{2} / \mathrm{Al}_{2} \mathrm{O}_{3}=4,24 \mathrm{~h}$ at $90{ }^{\circ} \mathrm{C}$; e,f) $\mathrm{SiO}_{2} / \mathrm{Al}_{2} \mathrm{O}_{3}=3,48 \mathrm{~h}$ at $120{ }^{\circ} \mathrm{C}$.] 
Tabela III - Resultados de FRX (\% em massa) do produto sintetizado por $48 \mathrm{~h}$ a $90{ }^{\circ} \mathrm{C}$ para $\mathrm{SiO}_{2} / \mathrm{Al}_{2} \mathrm{O}_{3}=3$. [Table III - XRF results (wt\%) of the product synthesized for $48 \mathrm{~h}$ at $90^{\circ} \mathrm{C}$ using $\mathrm{SiO}_{2} / \mathrm{Al}_{2} \mathrm{O}_{3}=3$. ]

\begin{tabular}{cccccc}
\hline $\mathrm{SiO}_{2}$ & $\mathrm{Al}_{2} \mathrm{O}_{3}$ & $\mathrm{Na}_{2} \mathrm{O}$ & $\mathrm{Fe}_{2} \mathrm{O}_{3}$ & $\mathrm{TiO}_{2}$ & P.F. \\
\hline 37,71 & 29,74 & 21,35 & 0,58 & 0,51 & 10,11 \\
\hline
\end{tabular}
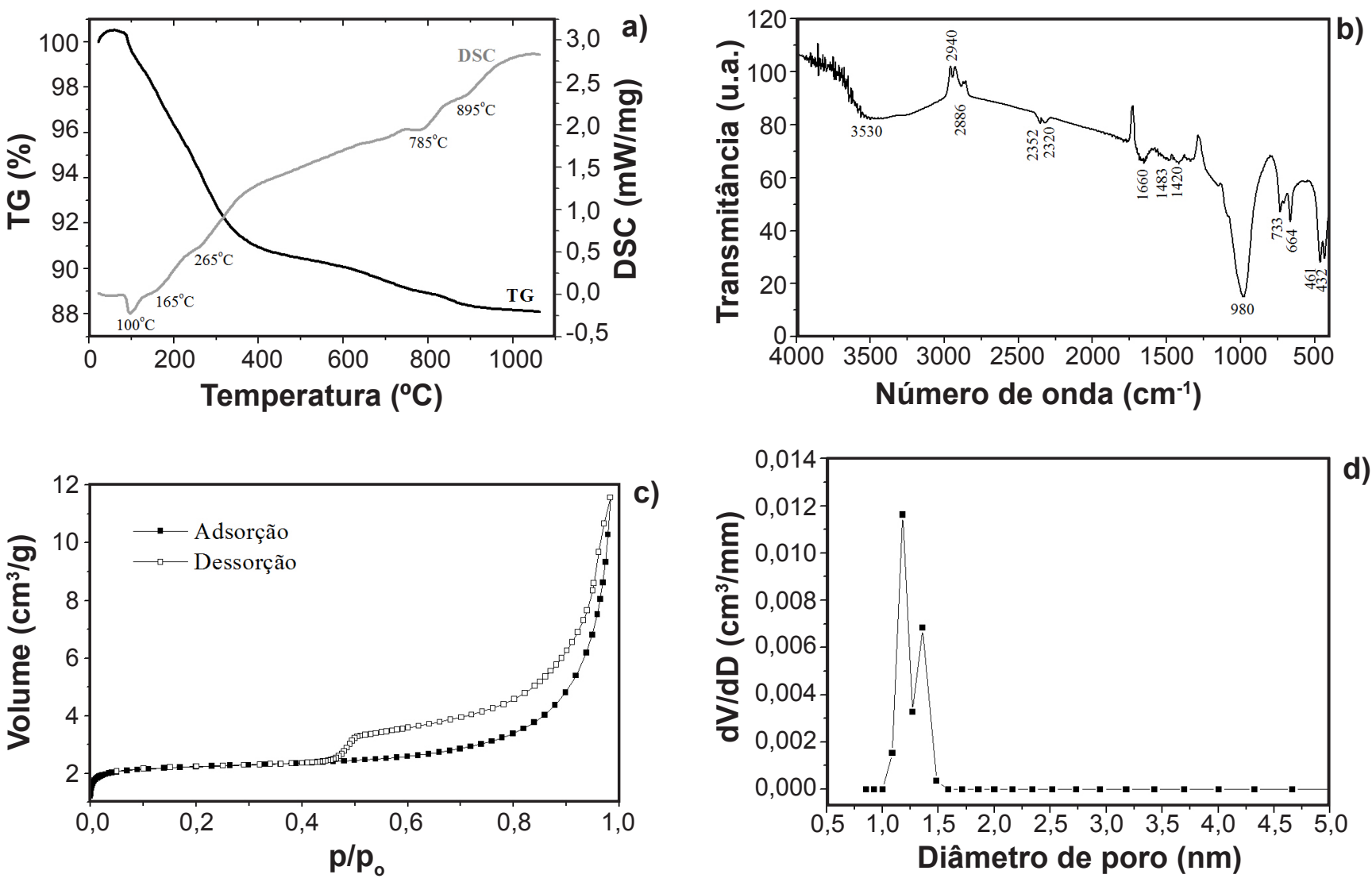

Figura 7: Resultados de caracterização do produto sintetizado por $48 \mathrm{~h}$ a $90{ }^{\circ} \mathrm{C}$ para $\mathrm{SiO}_{2} / \mathrm{Al}_{2} \mathrm{O}_{3}=3$ : a) curvas de $\mathrm{TG}$ e $\mathrm{DSC}$; b) espectro de FTIR; c) isotermas de adsorção-dessorção de $\mathrm{N}_{2}$; e d) curva de distribuição de diâmetro de poros.

[Figure 7: Characterization results of the product obtained for $48 \mathrm{~h}$ at $90^{\circ} \mathrm{C}$ using $\mathrm{SiO}_{2} / \mathrm{Al}_{2} \mathrm{O}_{3}=3:$ a) TG/DSC curves; b) FTIR spectrum; c) $\mathrm{N}_{2}$ adsorption-desorption isotherms; and d) pore diameter distribution curve.]

reações com alta alcalinidade $[15,24]$, como a estudada neste trabalho. Na literatura, estudos envolvendo sínteses de sodalita e cancrinita também revelaram a cancrinita como fase interferente à sodalita nas faixas de 120 a $140{ }^{\circ} \mathrm{C}$ [15] e 190 a $230^{\circ} \mathrm{C}$ [20]. A cancrinita e a sodalita são duas fases estáveis, sendo a fase cancrinita formada por meio da dissolução da sodalita e posterior precipitação sob a forma de zeólita cancrinita [25]. A semelhança estrutural resulta até na dificuldade na distinção dos picos ou quantificação de fases em análises de DRX [25].

Microscopia eletrônica de varredura (MEV): as análises morfológicas por MEV do ensaio de 48 h estão apresentadas nas Figs. 6a e 6b. Observou-se a ocorrência de formas arredondadas dos cristais, que cresceram e se aglomeraram como 'novelos de lã', característicos da zeólita sodalita $[9,26]$. Na imagem de MEV para o ensaio de 72 h (Fig. 6c), a morfologia da sodalita não ficou bem definida, não sendo possível observar de forma tão nítida os aglomerados. Uma microestrutura interessante foi obtida no ensaio de $\mathrm{SiO}_{2} / \mathrm{Al}_{2} \mathrm{O}_{3}$ igual a 4 (Fig. 6d), no qual a morfologia piramidal indicou ser de zeólita $\mathrm{X}$ (tipo faujasita), sendo possível observar os macroporos formados pelo empilhamento dos cristais. Zeólitas do tipo faujasita apresentam poros grandes, correspondendo à maior abertura entre as zeólitas citadas (7,4 Å) [4]. De acordo com as imagens de MEV para a temperatura de cristalização de $120{ }^{\circ} \mathrm{C}$, foi possível observar a formação da fase cancrinita, identificada pela presença de bastões (Fig. 6e) [20]. A fase sodalita também ainda estava presente, representada pelos cristais aglomerados em formato esférico (Fig. 6f) [20]. Em termos de maior cristalinidade e ausência de fases interferentes, o ensaio de $48 \mathrm{~h}$ de cristalização a $90{ }^{\circ} \mathrm{C}$ com razão $\mathrm{SiO}_{2} / \mathrm{Al}_{2} \mathrm{O}_{3}$ igual a 3 mostrou o melhor resultado em menos tempo, apresentando a sodalita como única fase cristalina, sendo possível observar por meio de MEV a morfologia bem definida desse tipo de zeólita. Portanto, a fim de caracterizar mais precisamente o produto zeolítico desse ensaio, foram realizadas análises de fluorescência de 
raios X (FRX), análises térmicas (TG/DSC), espectroscopia no infravermelho (FTIR), área específica BET e distribuição de diâmetro de poros.

Fluorescência de raios $X$ (FRX): os resultados da análise de FRX da amostra de sodalita sintetizada (48 h de cristalização a $90{ }^{\circ} \mathrm{C}$ com razão $\mathrm{SiO}_{2} / \mathrm{Al}_{2} \mathrm{O}_{3}$ igual a 3) estão apresentados na Tabela III. A análise química mostrou que $\mathrm{SiO}_{2}, \mathrm{Al}_{2} \mathrm{O}_{3}$ e $\mathrm{Na}_{2} \mathrm{O}$ foram os principais componentes do material com teores de $37,71 \%, 29,74 \%$ e $21,35 \%$ (\% em massa), respectivamente. $\mathrm{O}$ ensaio apresentou relação molar $\mathrm{SiO}_{2} / \mathrm{Al}_{2} \mathrm{O}_{3}$ igual a aproximadamente 2, valor condizente para a zeólita sodalita com baixo teor de silício apontado na literatura [4] e encontrado em outros trabalhos [9, 18]. Os óxidos $\mathrm{Fe}_{2} \mathrm{O}_{3}$ e $\mathrm{TiO}_{2}$ presentes podem estar relacionados com a não reação destes no processo, ou ainda pela substituição isomórfica de $\mathrm{Al}^{1+}$ e $\mathrm{Si}^{4+}$ por $\mathrm{Fe}$ e Ti na rede do aluminossilicato, porém outras técnicas seriam necessárias para tal averiguação.

Análise térmica (DSC/TG): os dados de análise térmica da sodalita (Fig. 7a) revelaram que a amostra sofreu perda de massa total de $12 \%$, quando aquecida desde a temperatura ambiente até acima de $1000{ }^{\circ} \mathrm{C}$. Uma perda ocorreu em torno de $100^{\circ} \mathrm{C}$ correspondendo à eliminação da água fisicamente adsorvida, observado pelo primeiro pico endotérmico na curva de DSC. A maior perda ocorreu até em torno de 400 ${ }^{\circ} \mathrm{C}$, quando um declive menos acentuado foi observado até a estabilização. A análise de DSC revelou evento endotérmico em torno de $165{ }^{\circ} \mathrm{C}$, representando a liberação de certa quantidade de moléculas de água das cavidades sodalita, o que resultou em uma estrutura parcialmente desidratada. Outro evento ocorreu na faixa de 250 a $400{ }^{\circ} \mathrm{C}$, quando a liberação de água chegou a resultar em uma estrutura completamente desidratada de sodalita, $\mathrm{Na}_{8}\left(\mathrm{AlSiO}_{4}\right)(\mathrm{OH})_{2}$ [15]. O pico observado em torno de $785^{\circ} \mathrm{C}$ referiu-se à decomposição da sodalita em $\alpha$-carnegieita, que em seguida transformou-se em nefelina, fase polimórfica mais densa $\left(\mathrm{NaAlSiO}_{4}\right)$, fenômeno representado pelo pico em torno de $895^{\circ} \mathrm{C}$ [15].

Espectroscopia no infravermelho (FTIR): no espectro vibracional no infravermelho da amostra de sodalita (Fig. $7 b$ ), foram identificadas as bandas em torno de 3000-3600 e $1660 \mathrm{~cm}^{-1}$ relacionadas com as espécies $\mathrm{OH}^{-}$e $\mathrm{H}_{2} \mathrm{O}$, respectivamente, hospedadas nas cavidades sodalita [27]. Foram identificadas ainda as bandas 733, 664, 461 e $432 \mathrm{~cm}$ ${ }^{1}$, estando em boa concordância com os picos para a zeólita sodalita relatados em trabalhos anteriores [28-30]. A banda intensa localizada em $980 \mathrm{~cm}^{-1}$ foi próxima a $1000 \mathrm{~cm}^{-1}$, que por sua vez foi atribuída às ligações $\mathrm{Si}-\mathrm{O}-\mathrm{Al}$ dos tetraedros $\mathrm{TO}_{4}$, características de materiais zeolíticos [29]. Esses resultados estão de acordo com outros autores que estudaram síntese de zeólita a partir de resíduo de caulim [17, 18, 27].

Caracterização textural por fisissorção de nitrogênio: o valor de área específica da amostra de sodalita sintetizada foi determinado pelo método BET com adsorção de nitrogênio. As isotermas de adsorção e dessorção de $\mathrm{N}_{2}$ são apresentadas na Fig. 7c. Foi observado que as isotermas apresentaram comportamento mais próximo ao do tipo IV, cuja característica principal consiste na existência de uma histerese associada normalmente à condensação capilar e na ausência de limitação de adsorção de $\mathrm{N}_{2}$ em valores elevados de pressão relativa $\left(\mathrm{p} / \mathrm{p}_{\mathrm{o}}\right)$. A histerese observada foi a do tipo $\mathrm{H} 3$, normalmente encontrada em materiais mesoporosos, consistindo em agregados ou aglomerados de partículas com formação de poros em forma de fenda (placas ou partículas com bordas, como cubos), com tamanho e/ou forma não uniformes. As zeólitas tipicamente pertencem a esta classe [31]. A área específica obtida pelo método BET foi de $8,5 \mathrm{~m}^{2} / \mathrm{g}$. Sodalitas formadas por síntese hidrotérmica apresentam valores de área específica próximos a $2 \mathrm{~m}^{2} / \mathrm{g}$ [32]. Entretanto, diferentes variáveis podem influenciar a área disponível, como as condições de síntese, tipos de ânions incorporados e presença de outras fases zeolíticas. Em outros trabalhos foram encontradas áreas específicas de 14,6 a $38,3 \mathrm{~m}^{2} / \mathrm{g}$ para sodalita com íons carbonato $\left(\mathrm{CO}_{3}{ }^{2-}\right)$ obtida a partir de resíduo de caulim [17], $33 \mathrm{~m}^{2} / \mathrm{g}$ para sodalita clorada sintetizada a partir de cinza volante [33] e 11,38 $\mathrm{m}^{2} / \mathrm{g}$ para sodalita obtida a partir de reagentes analíticos [34]. A Fig. 7d mostra a distribuição de diâmetro de poros para a amostra a partir de análise DFT. A amostra apresentou uma fração de microporos entre 1 e $1,5 \mathrm{~nm}$, indicando um material microporoso. Entretanto, como a área específica foi relativamente baixa $\left(8,5 \mathrm{~m}^{2} / \mathrm{g}\right)$, pode ter havido alguma obstrução dos poros da zeólita. Como é observado na Fig. $7 \mathrm{c}$, na isoterma houve uma inflexão na curva ligeiramente abaixo de $\mathrm{p} / \mathrm{p}_{\mathrm{o}}$ igual a 5,0 , devido ao fenômeno chamado de TSE (tensile strenght effect) [35]. Esse fenômeno ocorre quando há uma estrutura de poros maiores com gargalo menor que $4 \mathrm{~nm}$. Ou seja, a amostra possivelmente apresentou uma mesoporosidade ou macroporosidade (pela inflexão em altas pressões relativas), porém o acesso a esses poros foi pequeno. Outra possibilidade seria a formação de mesoporos do tipo secundário, gerados pelo empilhamento dos cristalitos da sodalita.

\section{CONCLUSÕES}

Para as condições de síntese estudadas, a relação sílica/alumina, o tempo e a temperatura de cristalização foram variáveis que influenciaram diretamente a formação de zeólita sodalita. Essa fase foi formada em melhores condições no tempo de $48 \mathrm{~h}$ de cristalização a $90^{\circ} \mathrm{C}$, usando relação sílica/alumina igual a 3 . Em outras condições, zeólitas A, X e cancrinita também foram formadas, existindo, assim, provável competição entre essas fases com a sodalita. Dessa forma, o controle das variáveis de processo torna-se indispensável para a obtenção do produto zeolítico desejado. A produção de sodalita a partir de resíduo de caulim com tratamento térmico mostrou-se, portanto, uma boa alternativa para o reaproveitamento desse tipo de material, o qual deixa de ser um poluente e passa a gerar um produto poroso com potencial utilização, por exemplo, em processos de separação de moléculas pequenas (como a água) presentes em misturas, na dessalinização de água do mar e outras soluções salinas, e na separação de gases em processos de combustão. 


\section{AGRADECIMENTOS}

Ao apoio do CNPq no Projeto PROCAD Amazônia 2018 - Processo número 88881.200618/2018-01 e pela bolsa de pesquisa.

\section{REFERÊNCIAS}

[1] A.B. Luz, A. Middea, $5^{\text {th }}$ UBC-McGill Bi-Annual Int. Symp. Fund. Miner. Proc. - Partic. Size Enlarg. Miner. Proc., Hamilton (2004) 22.

[2] A.B. Luz, A.R. Campos, E.A. Carvalho, L.C. Bertolino, R.B. Scorzelli, in "Rochas \& minerais industriais: usos e especificações", A.B. Luz, F.A.F. Lins (Eds.), CETEM/ MCT, Rio Janeiro (2008) 255.

[3] Depto. Nac. Prod. Mineral, http://www.dnpm.gov.br, ac. junho 2019.

[4] D.W. Breck, Zeolite molecular sieves: structure, chemistry, and use, John Wiley Sons, New York (1974).

[5] P. Souza Santos, Ciência e tecnologia de argilas, $2^{\mathrm{a}}$ ed., v.2, Edgard Blucher, S. Paulo (1989).

[6] S.M. Auerbach, K.A. Carrado, P.K. Dutta (Eds.), "Handbook of zeolite science and technology", Marcel Dekker, New York (2003).

[7] E.A. Hildebrando, R.S Angélica, R.F. Neves, F.R. Valenzuela-Diaz, Cerâmica 58, 348 (2012) 453.

[8] A.F. Gualtieri, P. Aprea, Micropor. Mesopor. Mat. 96 (2006) 276.

[9] S.P.A. Paz, R.S. Angélica, R.F. Neves, Quím. Nova 33, 3 (2010) 579.

[10] A.A. Maia, R.F. Neves, R.S. Angélica, H. Pöllmann, Clay Miner. 50 (2015) 663.

[11] C.G. Santos, P.M.P.B. Lana, A.A.S. Silva, C.N. Barbato, J.A. Sampaio, F.M.S. Garrido, F.A.N.G. Silva, Holos 28, 5 (2012).

[12] T. Thomsom, J. Nat. Philos. Chem. Arts 29 (1811) 285.

[13] L. Pauling, Z. Kristallogr. 74 (1930) 213.

[14] S. Khajavi, F. Kapteijn, J.C. Jansen, Membr. Sci. 63 (2007) 299

[15] S. Khajavi, S. Sartipi, J. Gascon, J.C. Jansen, F. Kapteijn, Micropor. Mesopor. Mat. 132 (2010) 510.

[16] L.B. McCuscker, F. Liebau, G. Engelhardt, Pure Appl. Chem. 73 (2003) 381.

[17] L.N. da Silva, "Desenvolvimento de processos de síntese de sodalita a partir de rejeitos de caulins da região Amazônica”, Diss. Mestr., Un. Fed. Pará, Belém (2013).
[18] A.A.B. Maia, E. Saldanha, R.S. Angélica, C.A.G. Souza, R.F. Neves, Cerâmica 53, 327 (2007) 319.

[19] R.S.F. Sena, "Estudo da síntese de zeólitas tipo A, X e sodalita empregando reatores vítreos: análise dos métodos dinâmico e estático de síntese a partir de caulim residual da Região Amazônica”, Diss. Mestr., Un. Fed. Pará, Belém (2013).

[20] F.A.C.M. Passos, J.A. Sampaio, A.A.S. Felix, F.M.S. Garrido, C.N. Barbato, F.A.N.G. Silva, in XXVI Enc. Nac. Tratam. Minér. Metal. Extrat. 26 (2015).

[21] F. Bergaya, B.K.G. Theng, G. Lagaly (Eds.), "Handbook of clay science", Elsevier, Amsterdam (2006).

[22] B.S. Carneiro, R.S, Angélica, T. Scheller, E.A.S. de Castro, R.F. Neves, Cerâmica 49, 312 (2003) 237.

[23] J. Cejka, H.V. Bekkum, A. Corma, F. Schuth (Eds.), "Introduction to zeolite science and practice", $3^{\text {rd }}$ ed., Elsevier, Amsterdam (2007).

[24] M. Maldonado, M.D. Oleksiak, S. Chinta, J.D. Rimer, J. Am. Chem. Soc. 135, 7 (2013) 2641.

[25] C.A.R. Reyes, C. Williams, O.M.C. Alarcón, Mater. Res. 16, 2 (2013) 424.

[26] C. Güther, H. Richter, I. Voigt, A. Michaelis, H. Tzscheutschler, R. Krause-Rehberg, J.M. Serra, Micropor. Mesopor. Mat. 214 (2015) 1.

[27] J.C. Buhl, W. Hoffmann, W.A. Buckermann, W. MüllerWarmuth, Solid State Nucl. Magn. Reson. 9 (1997) 121.

[28] M. Alkan, Ç. Hopa, Z. Yilmaz, H. Güler, Micropor. Mesopor. Mat. 86 (2005) 176.

[29] K. Zheng, A.R. Gerson, J. Addai-Mensah, R.S.C. Smart, J. Cryst. Growth 171 (1997) 197.

[30] E.M. Flanigen, H. Khatami, H.A. Szymanski, in "Molecular sieve zeolites-I", E.M. Flanigen, L.B. Sand (Eds.), Am. Chem. Soc. (1974) 201.

[31] G. Leofanti, M. Padovan, G. Tozzola, B. Venturelli, Catal. Today 41 (1998) 207.

[32] W. Ji, M. Li, C. Zeng, J. Yao, L. Zhang, Micropor. Mesopor. Mat. 143 (2011) 189.

[33] W. Franus, M. Wdowin, Environ. Monit. Assess. 186 (2014) 5721.

[34] K.K. Kaminishikawahara, L.J. Pereira, É.C.F. de Souza, M. Elena, P. Arrúa, S. Regina, M. Antunes, A.V.C. de Andrade, H.J. Alves, A.C. Antunes, Semin. Ciênc. Exatas Tecnol. 36, 2 (2015) 71.

[35] J.C. Groen, L.A.A. Peffer, P. Javier, Micropor. Mesopor. Mat. 60 (2003) 1.

(Rec. 25/03/2019, Rev. 15/06/2019, 01/12/2019, 15/05/2020, Ac. 18/05/2020) 